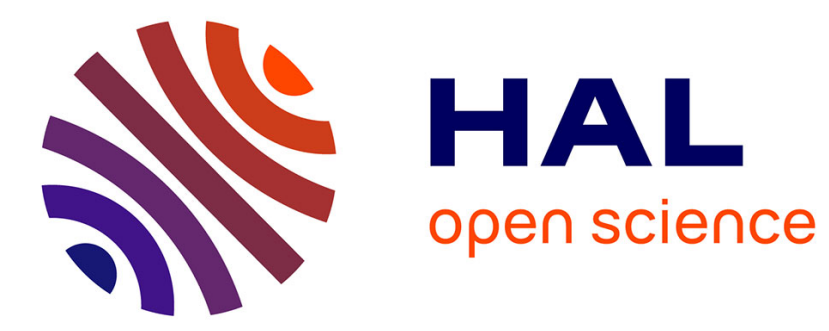

\title{
Robust statistical registration of 3D ultrasound images using texture information
}

François Rousseau, Ronan Fablet, Christian Barillot

\section{To cite this version:}

François Rousseau, Ronan Fablet, Christian Barillot. Robust statistical registration of 3D ultrasound images using texture information. ICIP 2003: IEEE International Conference on Image Processing, Sep 2003, Barcelone, Spain. pp.581 - 584, 10.1109/ICIP.2003.1247028 . hal-02341744

\section{HAL Id: hal-02341744 \\ https://hal.science/hal-02341744}

Submitted on 31 Oct 2019

HAL is a multi-disciplinary open access archive for the deposit and dissemination of scientific research documents, whether they are published or not. The documents may come from teaching and research institutions in France or abroad, or from public or private research centers.
L'archive ouverte pluridisciplinaire HAL, est destinée au dépôt et à la diffusion de documents scientifiques de niveau recherche, publiés ou non, émanant des établissements d'enseignement et de recherche français ou étrangers, des laboratoires publics ou privés. 


\title{
ROBUST STATISTICAL REGISTRATION OF 3D ULTRASOUND IMAGES USING TEXTURE INFORMATION
}

\author{
Rousseau François ${ }^{1}$, Ronan Fablet ${ }^{2}$ and Christian Barillot ${ }^{3}$ \\ ${ }^{1}$ IRISA, Université Rennes 1 \\ ${ }^{2}$ IFREMER/LASAA \\ ${ }^{3}$ IRISA, CNRS \\ Campus Beaulieu, Rennes \\ BP 70, 29280 Plouzané \\ Campus Beaulieu, Rennes \\ froussea@irisa.fr \\ rfablet@ifremer.fr \\ cbarillot@irisa.fr
}

\begin{abstract}
We investigate a new registration method for ultrasound volumes relying on on a statistical texture-based similarity measure. Texture information is given by spatial Gabor filters and represented by statistical kernel-based distributions. The registration similarity measure is then defined as a probabilistic distance, derived from Bhattacharyya coefficient, between two statistical distributions. Given this similarity measure, parametric ultrasound image registration is stated as a robust minimization issue. We also exploit frequency properties of spatial Gabor filters to propose a multiresolution approach to perform this minimization. We provide a preliminary evaluation of the new registration technique on clinical data.
\end{abstract}

\section{INTRODUCTION}

3D Registration of monomodality medical images is of key interest to visualize and quantify temporal changes in anatomy and physiology. Image registration has more particularly considered for brain imaging issues. However, whereas ultrasound imagery is well adapted for other body parts (throat, abdomen, heart,...), only few studies dealt with ultrasound image registration, since this type of image is of weak interest for brain imaging. Besides, the extension of registration techniques developed in the latter context is not straightforward due to low-quality ultrasound images for nonrigid organs. In addition, ultrasound is intrinsically a 2D measure. These different factors undoubtedly explain the lack of investigation concerning ultrasound image registration.

As it is mostly non-invasive and has a real time capability and a relatively low cost nature, 2D ultrasound is popular. Its major drawback is its weak capability of issuing quantitative accurate morphometric information [1]. In fact, conventional ultrasound exams are limited by $2 \mathrm{D}$ viewing, and follow-up studies are then not easily reproducible. 3D ultrasound imaging overcomes these limitations. In addition, it facilitates extensive investigation and allows accurate measurements of organ volumes.
The registration issue is to find the best alignment between two volumes. One of the volumes is chosen as the reference volume, and the other is transformed iteratively until the optimal alignment between the two data sets is found. Registration is required by ultrasound examinations for two main reasons. First, the combination of several volumes, called spatial compounding, can significantly improve the contrast-to-speckle noise ratio. Second, the registration of different ultrasound data set permits the comparison of serial examinations performed on the same patient.

Medical imaging techniques for image-based registration mainly rely on internal anatomic point, contour and surface landmarks, or voxel similarity. Internal landmark based registration techniques are limited since they require a specific segmentation. Contour and surface based registration methods also rely on accurate segmentation of anatomical structures. However, due to poor quality of ultrasound images, segmentation of ultrasound volumes is a very difficult task. Hence, voxel similarity-based methods seem to be more suited to ultrasound volume registration. As they require no segmentation, they are expected to be fully automatic.

A few voxel similarity-based methods have been proposed for ultrasound registration. Three main similarity measures were used: mutual information measure ([2]), correlation coefficient on intensity values [3] or on gradient images [4], and intensity values using optical flow hypothesis [5]. While ultrasound images are of relatively poor quality, they are highly textured. Besides, texture information has been proven to characterize ultrasound images, as highlighted for ultrasound image segmentation $[6,7]$.

As a consequence, it is highly attractive to consider on texture information for ultrasound image registration. In this paper, we investigate such an approach. Texture information is handled by means of spatial Gabor filters, which were shown to provide an accurate texture-characterization for texture classification and segmentation $[8,9]$. Texture information is rather a region feature than a pixel one. Thus, texture characteristics supplied by Gabor filters will be analyzed using local kernel-based density estimation [10]. Rigid 
ultrasound image registration is then stated as a robust multiresolultion minimization issue relying on a statistical similarity measure defined between the considered texture-based probabilistic distributions.

The paper is organized as follows. Section 2 presents texture features extracted by spatial Gabor filters. Section 3 introduces the statistical similarity measure exploiting nonparametric kernel density estimation. The registration algorithm is described in Section 4. Experiments are given in Section 5, and concluding remarks in Section 6 .

\section{TEXTURE FEATURE EXTRACTION}

In order to extract texture features from ultrasound volumes, we use a dyadic Gabor filter bank $([9,8])$. Such texture features were successfully used for ultrasound image segmentation with success in [7]. The impulse response of an even-symmetric Gabor filter is given by:

$$
\begin{aligned}
h(x, y, z)= & e^{-\frac{1}{2}\left(\frac{x^{2}}{\sigma_{x}^{2}}+\frac{z^{2}}{\sigma_{z}^{2}}+\frac{z^{2}}{\sigma_{z}^{2}}\right)} \\
& \times \cos \left(2 \pi\left(f_{x_{0}} x+f_{y_{0}} y+f_{z_{0}} z\right)\right)
\end{aligned}
$$

where $\left(f_{x_{0}}, f_{y_{0}}, f_{z_{0}}\right)$ is the center frequency of the filter, $\sigma_{x}$, $\sigma_{y}$ and $\sigma_{z}$ are the space constants of the Gaussian envelope along the $x, y$ and $z$ axes, respectively. Other orientations can be obtained by rotating the reference coordinate system.

As [9] suggested, five radial frequencies and four orientations have to be used in 2D with $f \in\left\{\frac{\sqrt{2}}{2^{6}}, \frac{\sqrt{2}}{2^{5}}, \frac{\sqrt{2}}{2^{4}}, \frac{\sqrt{2}}{2^{3}}, \frac{\sqrt{2}}{2^{2}}\right\}$. In 3D, we use thirteen orientations : $\theta \in\left\{0, \frac{\pi}{4}, \frac{\pi}{2}, \frac{3 \pi}{2}\right\}$ and $\phi \in\left\{0, \frac{\pi}{4}, \frac{\pi}{2}, \frac{3 \pi}{2}\right\}, \theta$ and $\phi$ are the rotation angles around the $z$ and $y$ axes respectively.

\section{SIMILARITY MEASURE}

We present in this section the statistical texture-based similarity measure exploited to formulate the registration issue between the two ultrasound volumes. We will in particular rely on kernel-based density estimator and on the Battacharrya as investigated by [10] for color image segmentation and filtering.

\subsection{Multivariate Kernel Density Estimate}

Let $\left\{\mathbf{x}_{i}\right\}_{i}$ be a set of $n$ points in $R_{d}$, a $d$-dimensional space. We denote by $\hat{f}(\mathbf{x})$ the multivariate density kernel estimate computed at point $\mathbf{x}$ as follows:

$$
\hat{f}(\mathbf{x})=\frac{1}{n h^{d}} \sum_{i=1}^{n} K\left(\frac{\mathbf{x}-\mathbf{x}_{i}}{h}\right)
$$

where $K(\mathbf{x})$ is the kernel and $h$ the window radius. The optimal kernel yielding minimal mean integrated square error is the multivariate Epanechnikov kernel. Its expression is given by:

$$
K_{E}(\mathbf{x})=\left\{\begin{array}{lr}
\frac{1}{2} c_{d}^{-1}(d+2)\left(1-\|\mathbf{x}\|^{2}\right) & \text { if }\|\mathbf{x}\|<1 \\
0 & \text { otherwise }
\end{array}\right.
$$

where $c_{d}$ is the volume of the unit $d$-dimensional sphere.

\subsection{Texture Representation}

As stated previously, texture is rather a region information. Thus, we exploit kernel density estimation to represent at a given point texture information provided by spatial Gabor filters. Let $\left\{r_{i}\right\}_{i=1, \cdots, n_{h}}$ be the voxel locations centered at $\mathbf{r}$ in a ultrasound volume. Given a texture value $u$, the associate probability in voxel $\mathbf{r}$ is given by:

$$
\hat{p}_{u}(\mathbf{r})=C_{h} \sum_{i=1}^{n_{h}} K\left(\frac{\mathbf{r}-\mathbf{r}_{\mathbf{i}}}{h}\right) \delta\left(u-V\left(\mathbf{r}_{\mathbf{i}}\right)\right)
$$

where $C_{h}$ is the normalization constant, $\delta$ is the Kronecker delta function. Texture value $u$ is in fact a vector of texture feature values, $V\left(\mathbf{r}_{\mathbf{i}}\right)$ is the texture feature vector for the voxel $\mathbf{r}_{\mathbf{i}}$. The resulting density $\hat{p}$ is the discrete estimated density using an histogram formulation. The further the voxels from the considered voxel $\mathbf{r}$, the lower weights they are assigned by kernel $K$. This property increases the robustness of the estimation.

\subsection{Bhattacharrya Coefficient}

Given the evaluation of statistical texture-based distributions, we define a probabilistic similarity measure between registered voxels using the the Bhattacharyya coefficient. This quantity is widely used, in particular for computer vision applications [10], to evaluate distance between probabilistic distributions. Given such two distributions $p$ and $q$, for the voxels $\mathbf{r}$ and $\mathbf{r}^{\prime}$ respectively, the Bhattacharyya coefficient $\rho\left(p(\mathbf{r}), q\left(\mathbf{r}^{\prime}\right)\right)$ is defined as:

$$
\rho\left(p(\mathbf{r}), q\left(\mathbf{r}^{\prime}\right)\right)=\int \sqrt{p_{\mathbf{z}}(\mathbf{r}) q_{\mathbf{z}}\left(\mathbf{r}^{\prime}\right)} d \mathbf{z}
$$

where $\mathbf{z}$ is the feature representing here texture information. The larger the coefficient, the more similar the two distributions.

Spatial Gabor filters provide at each voxel a texture feature vector of high dimension (typically, more than 60). Since kernel-based estimator are known to fail for high-dimensional space, we assume texture features at different scales and orientations are statistically independent. This assumption is also widely used when Gabor features are exploited for segmentation or classification purposes. Given this assumption, 
the global texture-based density is written as:

$$
p_{\mathbf{z}}(\mathbf{r})=\prod_{i=1}^{n_{g}} p_{z_{i}}(\mathbf{r})
$$

where $n_{g}$ is the size of a texture feature vector, $z_{i}$ is the $i^{\text {eme }}$ response given by the Gabor filter bank. Thus, each Gabor texture feature is analyzed separately, and associated to one-dimensional probabilistic density. The Bhattacharyya coefficient is now given by:

$$
\rho\left(p(\mathbf{r}), q\left(\mathbf{r}^{\prime}\right)\right)=\prod_{i=1}^{n_{g}} \rho\left(p_{z_{i}}(\mathbf{r}), q_{z_{i}}\left(\mathbf{r}^{\prime}\right)\right)
$$

From the Bhattacharyya coefficient, the similarity measure between two voxels the voxels $\mathbf{r}$ and $\mathbf{r}^{\prime}$ is finally defined as:

$$
d\left(\mathbf{r}, \mathbf{r}^{\prime}\right)=1-\rho\left(p(\mathbf{r}), q\left(\mathbf{r}^{\prime}\right)\right)
$$

This similarity measure depicts a rich texture information in a well-formalized probabilistic framework, and will be straightforwardly exploited for rigid ultrasound registration as explained in the following Section.

\section{ROBUST RIGID REGISTRATION}

In this section, we present our robust multiresolution framework for rigid ultrasound registration. We first introduce the parametric deformation model to be estimated. We will then state the registration issue as a robust minimization based on the statistical texture-based similarity measure. We will finally discuss its implementation within an incremental framework.

\subsection{Parametric Deformation Model}

We consider a generalized affine transformation involving the combination of four different transformations: scaling, shearing, rotation and translation. We use an affine parametric model with 12 parameters:

$$
v_{\theta}(\mathbf{r})=A(\mathbf{r}) \theta
$$

where $v_{\theta}(\mathbf{r})$ is the displacement vector for the voxel $\mathbf{r}=$ $\left(x_{r}, y_{r}, z_{r}\right)$ :

$$
v_{\theta}(\mathbf{r})=\left(\begin{array}{c}
a_{0}+a_{3} x_{r}+a_{4} y_{r}+a_{5} z_{r} \\
a_{1}+a_{6} x_{r}+a_{7} y_{r}+a_{8} z_{r} \\
a_{2}+a_{9} x_{r}+a_{10} y_{r}+a_{11} z_{r}
\end{array}\right)
$$

and $\theta=\left(a_{0}, \cdots, a_{11}\right)^{T}$, a vector of 12 parameters which have to be estimated.

\subsection{Robust formulation}

Solving for the optimal alignment between two 3D ultrasound volumes is stated as the recovery of the model parameters $\theta$ which minimizes a distance computed over the whole set of voxels of the transformed volume. The considered global distance is simply taken as the sum of the local probabilistic texture-based similarity measure introduce previously. In addition, M-estimators are considered to handle outliers during the minimization scheme ([5]). More precisely, the registration issue comes to solve for:

$$
\widehat{\theta}=\arg \min _{\theta} \sum_{\mathbf{r}} \alpha\left(1-\rho\left(p(\mathbf{r}), q\left(\mathbf{r}+v_{\theta}(\mathbf{r})\right)\right)\right.
$$

where $\alpha$ is the M-estimator. Robust M-estimation is equivalently formulated as an alternate weighted least-square minimization:

$$
\widehat{\theta}=\arg \min _{\theta} \sum_{\mathbf{r}} w_{r} \| 1-\rho\left(p(\mathbf{r}), q\left(\mathbf{r}+v_{\theta}(\mathbf{r})\right) \|^{2}\right.
$$

$w_{r}$ is an auxiliary variable acting as "weight", computed from the influence function $\beta(x)=\alpha^{\prime}(x) / x$ associated to considered M-estimator $\alpha$ ([5]). The M-estimation involves two steps. First, the computation of the robust weights for given residual errors, and, secondly, the weighted least-square minimization for given weights. In our experiments, we exploit the Cauchy estimator as M-estimator.

\subsection{Incremental Estimation}

We adopt an incremental strategy to solve for each weighted least-square minimization. Given a current estimate $\hat{\theta}_{k}$, it comes to estimate a small increment such that: $\theta=\hat{\theta}_{k}+$ $\Delta \theta_{k}$. This incremental strategy then involves a linearization of the quadratic criterion around the current solution in order to derive a close-form solution of the increment estimate.

More precisely, The linearization of the Bhattacharrya coefficient is given by:

$\rho\left(p(\mathbf{r}), q\left(\mathbf{r}+v_{\theta+\Delta \theta}(\mathbf{r})\right)=\rho\left(p(\mathbf{r}), q\left(\mathbf{r}+v_{\theta}(\mathbf{r})\right)+B^{T} . \Delta \theta\right.\right.$

where $B=\left.\frac{d \rho\left(p(\mathbf{r}), q\left(\mathbf{r}+v_{\theta+\Delta \theta}(\mathbf{r})\right)\right.}{d \Delta \theta}\right|_{\Delta \theta=0}$. After few manipulations, the incremental solution of (11) is:

$\hat{\Delta \theta}=\left(\sum_{\mathbf{r}} w_{\mathbf{r}} B B^{T}\right)^{-1} \sum_{\mathbf{r}} w_{\mathbf{r}}\left(1-\rho\left(p(\mathbf{r}), q\left(\mathbf{r}+v_{\theta}(\mathbf{r})\right)\right)\right) B$

with

$$
B=\frac{C_{h}}{h^{2}} \sum_{\mathbf{r}_{i}} p_{i}(\mathbf{r})\left(\frac{\mathbf{r}+v_{\theta}(\mathbf{r})-\mathbf{r}_{i}}{h^{2}}\right)^{T} \cdot A(\mathbf{r})
$$




$$
\begin{aligned}
p_{i}(r)= & \sum_{j=1}^{n_{g}}\left(\prod_{k \neq j} \sum_{c=1}^{n_{h}} \sqrt{p_{u_{k}^{c}}(\mathbf{r}) q_{u_{k}^{c}}\left(\mathbf{r}+v_{\theta+\Delta \theta}(\mathbf{r})\right)}\right) \\
& \times \sum_{c=1}^{n_{h}} \sqrt{\frac{p_{u_{j}^{c}}(\mathbf{r})}{q_{u_{j}^{c}}\left(\mathbf{r}+v_{\theta+\Delta \theta}(\mathbf{r})\right)}} \cdot \delta\left(u_{j}^{c}-V_{j}^{c}\left(\mathbf{r}_{i}\right)\right)
\end{aligned}
$$

This incremental minimization is stopped when the increment estimate is beyond a given threshold.

In addition, we exploit the characteristics of Gabor features to conduct this robust registration within a multiresolution framework. In fact, the minimization is initiated with low resolution Gabor features, while higher resolution $\mathrm{Ga}-$ bor features are incrementally introduced during the minimization.

\section{EXPERIMENTS}

Registration was applied to clinical ultrasound volumes of carotid. Affine transformations have been applied on these volumes. We have created an artificially deformed volume by using a Thin Plate Spline deformation. This produces a global smooth deformation which simulate non-rigid motion in tissues. Thus, the proposed method can be evaluated qualitatively and quantitatively.

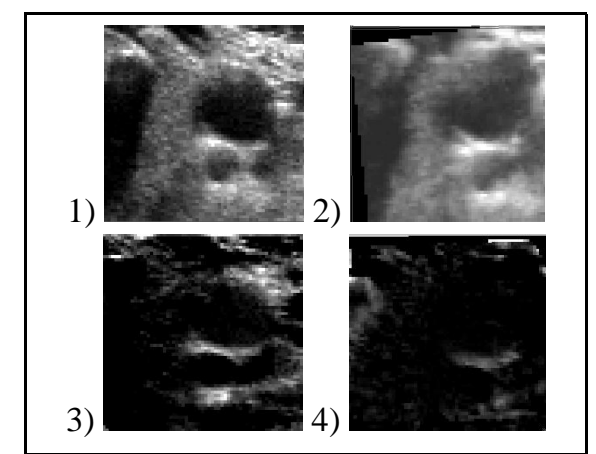

Fig. 1. Carotid Ultrasound Volumes. 1) original volume, 2) deformed volume, 3) difference between original and deformed volume, 4) difference original and registered volume.

To evaluate the preliminary results, we compute the mean error $\left(e_{1}, e_{2}\right)$ concerning the translation vector and the matrix including rotation, shearing and scaling transformations using the Frobenius norm: $\left(e_{1}, e_{2}\right)=(0.47,0.12)$. Moreover, as a qualitative measure, the difference image between the original volume and the registered one has been chosen (Figure 1). More experiments have to be done to validate the proposed method.

\section{CONCLUSION}

We have presented a new registration algorithm for ultrasound volumes that includes texture information using a sta- tistical similarity measure. Texture feature vectors obtained with a Gabor filter bank, have been represented by kernelbased distributions. A robust parametric registration algorithm have been proposed, using also the multiresolution properties of Gabor filters. Preliminary promising results have been presented. Further investigations are needed, more experiments will be done to validate the proposed registration method.

\section{REFERENCES}

[1] A. Fenster, D. B. Downey, and H. N. Cardinal, "Threedimensional ultrasound imaging," Physics in medicine and biology, vol. 46, pp. 67-99, 2001.

[2] R. Shekhar and V. Zagrodsky, "Mutual imformationbased rigid and nonrigid registration of ultrasound volumes," IEEE Trans. Medical Imaging, vol. 21, no. 1, pp. 9-22, 2002.

[3] G. Xiao, M. Brady, J. A. Noble, M. Burcher, and R. English, "Nonrigid registration of 3-d free-hand ultrasound images of the breast," IEEE Trans. Medical Imaging, vol. 21, no. 4, pp. 405-412, 2002.

[4] R. Rohling, A. Gee, and L. Berman, "Automatic registration of 3d ultrasound images," Ultrasound in Medicine and Biology, vol. 24, pp. 841-854, 1998.

[5] I. Pratikakis, C. Barillot, and P. Hellier, "Robust multiscale non-rigid registration of $3 \mathrm{~d}$ ultrasound images," in Int. Conf. on Scale-Space and Morphology in Computer Vision, 2001, pp. 389-397.

[6] D. Boukerroui, O. Basset, A. Baskurt, and A. Noble, "Segmentation of echocardiographic data. multiresolution $2 \mathrm{~d}$ and $3 \mathrm{~d}$ algorithm based on gray level statistics," in Proc. of Medical Image Computing and Computer-Assisted Intervention, 1999, pp. 516-524.

[7] C-M Chen, H. H-S Lu, and K-C Han, "A textural approach based on gabor functions for texture edge detection in ultrasound images," Ultrasound in Medicine and Biology, vol. 27, no. 4, pp. 515-534, 2001.

[8] D. Dunn, W. Higgins, and J. Wakeley, "Texture segmentation using 2-d gabor elementary functions," IEEE Trans. Pattern Analysis and Machine Intelligence, vol. 16, no. 2, pp. 130-149, 1994.

[9] A.K. Jain and F. Farrokhnia, "Unsupervised texture segmentation using gabor filters," Pattern Recognition, vol. 24, no. 12, pp. 1167-1186, 1991.

[10] D. Comaniciu and P. Meer, "Mean shift : a robust approach toward feature space analysis," IEEE Trans. Pattern Analysis and Machine Intelligence, vol. 24, no. 5, pp. 1-18, 2002. 